\title{
A case of meconium peritonitis due to bowel perforation diagnosed at anomaly scan at 20 weeks
}

\author{
Prabha Sinha', Shabnum Sibtain², Hakmi Aoun ${ }^{3}$ \\ 'Ms, FRCOG, MRCPI, Diploma in Advanced Obstetrics Ultrasound RCOG/RCR, Dip Mgmt, Dip Med Education, \\ DGO, MBBS, Consultant Tawam Hospital, Abu Dhabi, UAE \\ ${ }^{2} \mathrm{Ms}$, FRCOG, MRCOG, DRCOG, MBBS, Asst Prof Azra Naheed Medical college, Lahore. Pakistan \\ ${ }^{3}$ Consultant Tawam Hospital, Abu Dhabi, UAE
}

\section{Corresponding Author}

Shabnum Sibtain,16 Empress Road, Lahore, Tel.: +923344201333, e-mail: s_sibtain@hotmail.com

\begin{abstract}
Meconium peritonitis is a rare condition in a fetus which occurs due to perforation of the bowel in the antenatal period. It has serious neonatal consequences resulting in death if the diagnosis is delayed soon after delivery. However, its detection is difficult as the presentation is very variable. A case of meconium peritonitis and pseudocyst detected in a fetus at 33 weeks and 2 days of gestation is discussed regarding its diagnosis and management. Final diagnosis was made only after delivery and managed by surgery (bowel resection). The outcome after treatment was favourable because of the timely intervention.
\end{abstract}

Key words: Perforation of bowel in neonates, prenatal diagnosis, neonatal ascites

\section{Introduction}

It is possible to diagnose both meconium peritonitis and meconium pseudo-cysts antenatally by ultrasound where bowel appears to be dilated with moderate to severe ascites. These anomalies especially with massive ascites can cause fetal hypoxia in antenatal period due to compression of the chest. Therefore such cases require vigilance and referred to the tertiary center with a specialized unit with the facilities of paediatric surgery to manage this condition. After the delivery abdominal X-ray is recommended where meconium peritonitis and meconium pseudo-cysts is suspected. On X-ray it may present as a calcified or cystic abdominal mass with multiple air fluid levels. Meconium peritonitis presenting with meconium pseudo-cysts is not commonly seen. In this condition meconium leaks to the peritoneal cavity from the bowel and causes cyst meconium peritonitis which has fibrous wall. However meconium pseudocyst has smooth muscle layer connecting the cyst to normal intestine. The lack of epithelium happens due to inflammation. Both meconium peritonitis cystic type and meconium pseudocyst can only be differentiated on histopathologic findings. ${ }^{1}$ 
Case report: G5 P3+1, all preterm delivery by caesarean section (CS) at 25 and 28 and 31 weeks - for abruption, preterm pre-labour rupture of membranes and preterm labour respectively. Her Last CS was in September 2014. She had history of bariatric surgery cholecystectomy and non-obstructive pelvi-calyceal stone in the right kidney. She had cholestasis and was taking ursodeoxycholic acid. She was IgG positive for cytomegalovirus.

Routine anomaly scan done at 20 weeks showed dilated bowel with fetal ascites. Kidneys, four chambers of heart, spine and profile were not well seen. The differential diagnosis of Hirschsprung's disease and Down syndrome was made. She was referred to tertiary hospital with fetal medicine unit. At 26 weeks gestation on ultrasound there was minimal to moderate amount of ascites with a trace of pericardial effusion. Portal vein looked dilated and bowels also noted to be dilated. No other fetal anomaly noted. Her 32 weeks of gestation ultrasound showed much dilated bowel, fetal ascites had subsided but large cystic lesion in the abdomen was present, appeared to be large bowel. No pericardial effusion was noted. Placenta looked normal with succenturiate lobe. Normal liquor and Doppler's noted. Genetic cause for the cause could not be excluded.

She was delivered by emergency CS at $33+2$ wks. The weight of baby was $1.47 \mathrm{~kg}$. Due to abdominal distension and ultrasound antenatal findings further investigations were performed followed by laparotomy. Meconium pseudocyst, perforation in terminal ilium (10 cm from ileocecal valve), malrotation with small and large bowel dense adhesion were detected. Resection of necrosed bowel and Ileostomy was performed due to in-utero perforation of terminal ilium (10 cm from ileocecal valve). Baby had G6PD deficiency and reducible inguinal hernia, Short Bowel syndrome due to high ileostomy. Chromosomes were normal.

Baby was discharged after 4 weeks. On follow up baby was lagging behind the mile stones and had nutritional deficiency due to short gut syndrome but keeping reasonably well.

\section{Discussion}

Meconium peritonitis with meconium pseudocyst is a grave condition which results from bowel perforation leading to the death of the new born. The aetiology of the ileal perforation is unknown. There were no findings in the above mentioned case to suggest connective tissue disorder. In the meconium peritonitis the bowel perforation in the fetus, results in the leaking of meconium into the peritoneal cavity resulting in the inflammation of the peritoneum. The meconium pseudocyst is formed between intestine and omentum, in cases where the perforation closes spontaneously. ${ }^{2}$ In meconium peritonitis with pseudocyst, there is fetal abdominal mass with diverse echogenic and hyper echogenic calcifications on ultrasound. ${ }^{3}$ The ascites or polyhydramnios or both are commonly associated with it. Intra-uterine bowel perforation can occur secondary to a variety of abnormalities and cause sterile peritonitis in the fetus (generalized type1). If sealing of the perforation does not take place, a thick-walled pseudo-cyst can form (type II) which was the likely sequence of events in this case.

Those babies who have large abdominal cysts with air and fluid content, meconium peritonitis should be suspected. The number of pseudocyst diagnosed as calcified cyst on X-ray is more than $85 \%$ however the diagnosis can not only be made on the X-ray. The diagnosis has to be confirmed by the histopathology and to differentiate between the cystic form of meconium peritonitis and meconium pseudocyst. ${ }^{4}$ Both cystic type meconium peritonitis and meconium pseudocyst are treated by surgery. In cystic-type meconium peritonitis initially in most of the cases drainage is done followed by surgery. The recommendation regarding treatment of meconium 
pseudocyst, is intestinal resection with anastomosis. ${ }^{5}$

The advancements in ultrasound technology have increased the detection rate of meconium peritonitis in the fetus antenatally. There may be marked ascites in the fetus. This may cause hydrops and hypoplastic lungs in the fetus. Currently there is no consensus on the meconium peritonitis management in the antenatal period. Early recognition, surgical correction, antibiotics and meticulous postoperative care is the common practice to improve survival.

In order to prevent fetal hydrops and hypoplastic lungs, multiple paracentesis are done in case of marked ascites. If there is polyhydramnios, amniocentesis is advisable to prevent preterm labour. ${ }^{6}$ All these measures are likely to improve the outcomes.

\section{Conflict of interest}

Nothing to declare.

\section{Consent}

Verbal.

\section{References}

1. Minato M, Okada T, Miyagi H, Honda S, Takazawa K, Kubota KC, Todo S. Meconium pseudocyst with particular pathologic findings: a case report and review of the literature. J Pediatr Surg 2012 Apr;47(4):e9-12.
2. Markov D, Jekova N, Ivanov S, Diavolov V, Brankov O. Fetal meconium pseudocyst secondary to in utero perforation of colon transversum and meconium peritonitis. Akush Ginekol (Sofiia). 2011;50(1):46-51.

3. Dewan P, Faridi MM, Singhal R, Arora SK, Rathi V, Bhatt S, Aggarwal SK. Meconium peritonitis presenting as abdominal calcification: three cases with different pathology. Ann Trop Paediatr 2011;31(2):163-7.

4. Gabrielle Shu-yi Lee, Suresh Chandran, Victor Samuel Rajadurai. Calcified meconium pseudocyst: X-ray diagnosis of meconium peritonitis at birth. BMJ Case Rep 2015 Jul 7;2015:bcr2015211052.

5. Wang Y, Wu Y, Guan W, Yan W, Li Y, Fang J, Wang J Meconium peritonitis due to fetal appendiceal perforation: two case reports and a brief review of the literature. BMC Pediatr 2018 May 11;18(1):162.

6. Uchida, K, Koike, Y, Matsushita, K, Nagano, Y, Hashimoto, K, Otake, K, Inoue, M, \& Kusunoki, M. Meconium peritonitis: Prenatal diagnosis of a rare entity and postnatal management. Intractable Rare Dis Res 2015 May;4(2):93-7.

Received 23-07-20

Revised 03-09-20

Accepted 17-09-20 